\title{
Atlas of Pulmonary Vascular Imaging
}

\author{
C. Wittram
}

New York, NY: Thieme Medical Publishers, Inc., 2011, 161 pages, $\$ 99.95$

The depiction of pulmonary and systemic vessels in the thorax has improved dramatically as CT and MRI have continued to improve. The pulmonary vessels and their relation to other intrathoracic structures are demonstrated in ways impossible previously, with better spatial, contrast, and temporal resolutions, and thus diagnoses of many congenital and acquired diseases have been improved. Multidetector CT has revolutionized the diagnosis of acute pulmonary emboli.

This book illustrates a comprehensive and systematic approach to common, uncommon, and rare diseases of pulmonary vessels with clear radiographic, angiographic, ultrasound, CT, MRI, and nuclear medicine images. This lavish atlas teaches readers how to identify and quickly diagnose the spectrum of pulmonary vascular pathologies using various imaging modalities with detailed and labeled images.

The atlas is well organized into 12 sections with a pertinent bibliography, and the first section, on normal anatomy, sets the stage with excellent drawings and images of various modalities. Two sections follow on vascular changes in congenital lung and cardiac diseases. The remaining sections discuss specific disorders such as embolism, in situ thrombosis, aneurysm and varix, vasculitis, infection, trauma and intervention, tumors, systemic and lung diseases, and pulmonary arterial hypertension. Each concise yet comprehensive section provides systematic coverage of various disorders of the pulmonary vessels. Particularly high-quality angiographic and correlative CT images, as well as instructive drawings, point out the diagnostic criteria of pulmonary embolism. There are 322 high-resolution images in 188 figures, including 24 color ones, which make for easy understanding. Lists of suggested reading are provided for those who desire more indepth information.

This image-rich atlas with concise, important information is useful for clinicians, fellows, and residents in radiology, nuclear medicine, respiratory and emergency medicine, cardiology, and cardiothoracic surgery as both an educational tool and a user-friendly reference for everyday practice. It also provides a foundation of understanding and encourages interest in and further research of pulmonary vascular diseases. I highly recommend this atlas to all radiologists and nuclear physicians.

\author{
E. Edmund Kim \\ M.D. Anderson Cancer Center \\ 1515 Holcombe Blvd., Unit 1264 \\ Houston, TX 77030 \\ E-mail: ekim@mdanderson.org
}

COPYRIGHT @ 2011 by the Society of Nuclear Medicine, Inc. 